\title{
COMPARISON OF HAEMODYNAMIC PROFILE AFTER SPINAL ANAESTHESIA IN PATIENTS ON REGULAR TREATMENT WITH CALCIUM CHANNEL BLOCKERS AND $\beta$ BLOCKERS
}

\author{
Kavyashree N. G1, Muralidhara Danappa Patel2, Girish C. N3, Ravishankar R. B
}

${ }^{1}$ Assistant Professor, Department of Anaesthesiology, Shridevi Institute of Medical Sciences and Research Hospital, Tumkur, Karnataka. ${ }^{2}$ Assistant Professor, Department of Anaesthesiology, Shridevi Institute of Medical Sciences and Research Hospital, Tumkur, Karnataka. ${ }^{3}$ Assistant Professor, Department of Anaesthesiology, Shridevi Institute of Medical Sciences and Research Hospital, Tumkur, Karnataka. 4 Professor, Department of Anaesthesiology, JJM Medical College, Davangere, Karnataka.

\begin{tabular}{l}
\hline ABSTRACT \\
\hline BACKGROUND \\
Hypertensive patients develop wide swings in blood pressure intraoperatively, especially after spinal anaesthesia. Long term \\
antihypertensive agents can modify this effect by controlling blood pressure. This study was undertaken to evaluate the \\
haemodynamic effect in hypertensive patients on regular treatment with calcium channel blockers and beta-blockers who are \\
undergoing elective surgery under spinal anaesthesia and compared with normotensives.
\end{tabular}

AIMS

To study the intraoperative changes of blood pressure and heart rate in patients on calcium channel blockers and beta-blockers undergoing surgery under spinal anaesthesia and to compare the data with normotensive patients.

\section{MATERIALS AND METHODS}

90 patients were included in the study; 30 patients were normotensives (Group N) and 30 patients were hypertensives who were regularly on calcium channel blockers (Group C) and 30 patients on beta-blockers (Group B). Both the groups continued the drug on the day of surgery. The baseline blood pressure and heart rate were recorded. After spinal anaesthesia, the blood pressure and heart rate were noted at $2 \mathrm{~min}, 4 \mathrm{~min}, 6 \mathrm{~min}, 8 \mathrm{~min}, 10 \mathrm{~min}, 15 \mathrm{~min}$ and thereafter with 5 minutes interval till the end of the procedure.

\section{STATISTICAL ANALYSIS}

Baseline data was analysed using descriptive statistics. Intragroup variation in parameters analysed by one way ANOVA and within the different time intervals by post-hoc test. Intergroup comparison by unpaired ' $\mathrm{T}$ ' test. P value $<0.05$ considered statistically significant.

\section{RESULTS}

The hypotension was evident in Group C compared to other groups, but bradycardia (heart rate <60) more seen in Group B and were statistically significant $(\mathrm{P}<0.001)$. The usage of rescue medication was more in study group and was statistically significant $(\mathrm{P}<0.002)$.

\section{CONCLUSION}

Incidence of hypotension was more with patients on calcium channel blockers and required vasopressors and fluids, but incidence of bradycardia was seen more in patients on beta blockers who needed atropine. Anaesthesiologists should anticipate and be adequately prepared for any untoward consequences.

\section{KEYWORDS}

Hypertension, Spinal Anaesthesia, Calcium Channel Blockers, Hypotension, Beta Blockers, Bradycardia.

HOW TO CITE THIS ARTICLE: Kavyashree NG, Patel MD, Girish CN, et al. Comparison of haemodynamic profile after spinal anaesthesia in patients on regular treatment with calcium channel blockers and $\beta$ blockers. J. Evolution Med. Dent. Sci. 2016;5(34): 1922-1928, DOI: $10.14260 /$ jemds/2016/455

\section{INTRODUCTION}

Spinal anaesthesia is the most commonly used procedure for lower abdominal and limb surgeries and common side effects being hypotension and bradycardia due to sympathetic denervation after spinal anaesthesia. Reason being maximal venodilatation causing peripheral pooling and decreased preload to the heart, however, smooth muscles in arteries maintain autonomic tone after spinal also.

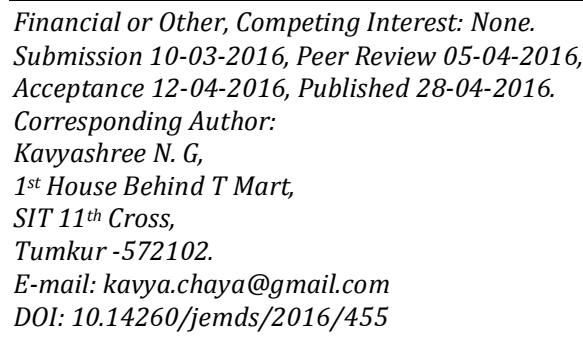

Hypertensive patients can develop wide swings in blood pressure intraoperatively, which increases the risk of postoperative cardiac and renal complications such as myocardial ischemia, cerebrovascular accidents and acute renal failure, independent risk factors.1,2 were identified which are necessary for the prevention of perioperative cardiac events causing both morbidity and mortality. Structural changes in arteriolar walls play a primary sole in haemodynamic response to anaesthesia and explain greater changes in systemic vascular resistance and arterial pressure in hypertensive patients than normotensive patients with similar degree of sympathetic blockade. ${ }^{3}$

Studies have been done to determine whether it is beneficial to continue Calcium channel blockers and Beta blockers. ${ }^{4}$ in patients undergoing spinal anaesthesia. Effect of continuation of angiotensinogen converting enzyme inhibitors (ACE I). ${ }^{5}$ in patients undergoing spinal anaesthesia and found 
that continuation of ACE I did not affect early hypotension severity compared to normal individuals. There is inadequate evidence on the effects of Calcium channel blockers and Beta blockers on Blood pressure in hypertensive patients undergoing Spinal anaesthesia. Because of end organ changes in hypertensive patients, post spinal hypotension could have detrimental effect.

So this study was an effort to compare the incidence of hypotension and bradycardia in hypertensive patients on regular medication with standardized doses of calcium channel blockers and beta-blockers with that in normotensive patients after spinal anaesthesia.

\section{MATERIALS AND METHODS}

The study was conducted on 90 patients. Group N (Normotensive group), Group C (Calcium channel blockers group), Group B (Beta blocker group) undergoing elective surgeries under spinal anaesthesia at Chigateri Government Hospital and Bapuji Hospital attached to JJM Medical College, Davangere, from 2013 to 2014.

\section{Inclusion Criteria}

Patients undergoing elective surgeries under spinal anaesthesia who are between 30 and 60 years old (ASA 1 and ASA 2) and diagnosed cases of essential hypertension who are on anti-hypertensive medications at least 1 month prior to surgery.

\section{Exclusion Criteria}

1. Patients having contraindications for spinal anaesthesia.

2. Patients with other co-existing diseases like diabetes, coronary artery disease or other cardiac diseases, severe hypovolemia, sepsis, pregnant patients.

The Institutional Ethical clearance was obtained before beginning the study. Informed written consent about procedure was obtained prior. During preanaesthetic evaluation, patients were assessed and explained about the anaesthetic procedure and peroperative monitoring. History regarding anti-hypertensive medications, duration of treatment, other co-existing diseases and any other medications were taken. In operating room, after inserting the 18G IV cannula patients were coloaded with $15 \mathrm{~mL} / \mathrm{kg}$ of Ringer Lactate (RL) solution, maintenance fluid continued throughout procedure. Total volume infused were recorded. Standard monitoring included continuous ECG, pulse rate, oxygen saturation with a pulseoximeter and automated NonInvasive Blood Pressure (NIBP) Systolic (SBP) and Diastolic (DBP) and Mean Blood Pressures.

Baseline values were taken Spinal anaesthesia was given in the left lateral position at L3-L4 interspace with all the aseptic precautions using a 25-gauge Quincke-Babcock spinal needle. After noticing a free flow of the cerebrospinal fluid, 3 $\mathrm{mL}$ of $0.5 \%$ hyperbaric bupivacaine was administered over a 10 second period. The patient was put in a supine position. The level of sensory blockade was determined by a pin-prick at 5 min and $15 \mathrm{~min}$ after the drug administration. NIBP and pulse rate were recorded every $2 \mathrm{~min}$ for the first $10 \mathrm{~min}$ and every $5 \mathrm{~min}$ till the end of the surgical procedure.

Hypotension was defined as a decrease of mean arterial pressure of more than $20 \%$ from baseline and was intervened with fluids or vasopressors within $20 \mathrm{~min}$. Mephentermine 6 $\mathrm{mg} /$ dose and IV fluids $5 \mathrm{~mL} / \mathrm{kg}$ were used for Hypotension until both Systolic Arterial Pressure (SAP) and Mean Arterial Pressure (MAP) increased above the threshold level. The time of rescue dose and the total dose for hypotension was recorded.

Bradycardia was defined as heart rate $<60$ beats/min and was treated with Inj. atropine $0.6 \mathrm{mg}$ if not responding to intravenous fluids.

\section{STATISTICAL ANALYSIS}

Baseline data was analysed using descriptive statistics. The changes of systolic blood pressure, diastolic blood pressure, mean arterial pressure and heart rate were analysed as follows:

1. The changes of the parameter within the group was analysed using one way ANOVA and the variation within the different time intervals was analysed with the posthoc tests.

2. Intergroup comparison at the $2 \mathrm{~min}, 4 \mathrm{~min}, 6 \mathrm{~min}, 8 \mathrm{~min}$, $10 \mathrm{~min}$ and 15 minutes from the data of controls and cases respectively was done by unpaired ' $\mathrm{t}$ ' test.

' $\mathrm{P}$ ' value $<0.05$ was considered significant and $<0.01$ highly significant.

\section{RESULTS}

\section{Demographic Data}

The demographic characteristics of both the groups were comparable as shown in Table 1.

\section{Average Sensory Block}

The average height of the block after spinal anaesthesia was at the 7th to 8th thoracic level Table 2 .

\section{Baseline Haemodynamics (Table 3)}

The average baseline systolic blood pressure was $134.53 \pm 5.42$ $\mathrm{mmHg}$ in group $\mathrm{C}$ and $135.93 \pm 4.91 \mathrm{mmHg}$ in group $\mathrm{B}$, which was on an average $6.5 \mathrm{mmHg}$ and $8 \mathrm{mmHg}$ higher in the hypertensive group $\mathrm{C}$ and group $\mathrm{B}$ respectively than the normotensive group. The baseline diastolic blood pressure was $82.93 \pm 6.54 \mathrm{mmHg}$ in group $\mathrm{C}$ and $80.73 \pm 9.23$ in group $\mathrm{B}$, which was on an average $6.86 \mathrm{mmHg}$ and $4.66 \mathrm{mmHg}$ higher in the hypertensive group $C$ and group $B$ respectively than the normotensive group. The baseline mean blood pressure was $100.18 \pm 5.95 \mathrm{mmHg}$ in group $\mathrm{C}$ and $99.27 \pm 7.41$ in group $\mathrm{B}$ which was on an average $6.8 \mathrm{mmHg}$ and $5.87 \mathrm{mmHg}$ higher in the hypertensive group $\mathrm{C}$ and group $\mathrm{B}$ respectively than the normotensive group. The baseline heart rate was almost the same in normotensive group $\mathrm{N}$ and the hypertensive group $\mathrm{C}$ (70 bpm) and it was on lower side in the hypertensive group $B$ (67 bpm).

Systolic blood pressure after spinal Anaesthesia (Table 4, Table 5, Fig. 1): Hypotension was observed in all groups, statistically significant fall in systolic blood pressure occurred from 4 minutes after spinal up to 15 minutes. Fall from baseline SBP (Table 4) in control group at 4, 6, 8, 10, 15 minutes were $16 \pm 8.2(\mathrm{mmHg}), 20.6 \pm 10.3(\mathrm{mmHg}), 20 \pm 11$ (mmHg), 21 \pm 10 (mmHg), $17.8 \pm 10.4$ (mmHg). In group $\mathrm{C}$ at 4 , $6,8,10,15$ minutes were $27 \pm 15 \mathrm{mmHg}, 30.27 \pm 17.28 \mathrm{mmHg}$, $28 \pm 18 \mathrm{mmHg}, 31 \pm 18 \mathrm{mmHg}, 27 \pm 16.95 \mathrm{mmHg}(\mathrm{P}<0.001)$ statistically significant fall in systolic blood pressure occurred compared to control group and group B. Fall in SBP was 
observed in group B at 4, 6, 8, 10, 15 minutes, but not significant from control group (Table 5).

Diastolic blood pressure and mean blood pressure (Table 6, Table 7, Fig. 2): Statistically significant fall in Diastolic Blood Pressure in group $C$ at 4, 6, 8, 10, 15 minutes compared to other two groups were $21.2 \pm 4.5,23.3 \pm 4.1,22.6 \pm 4.18$, $23.4 \pm 6.7,22.7 \pm 6.7 \mathrm{mmHg}$ from the baseline $(\mathrm{P}<0.001)$ (Table 6 and 7). Changes in mean arterial pressures showing significant fall at 4, 6, 8, 10,15 minutes observed more in group $\mathrm{C}$ (Table 8 and 9) Fig. 3, 14/30 patients in group $\mathrm{C}$ required Mephentermine for hypotension (Table 11).

Heart rate variation: Fall in heart rate observed significantly in group B compared to other groups at 2, 4, 6, 8, 10 , 15 minutes were $5.2 \pm 2.4,6.5 \pm 1.8,7.7 \pm 1.7,10.2 \pm 0.58$, $10.4 \pm 0.81, \quad 10.5 \pm 0.8$ rate/minute from the baseline (Table 10), 16/30 of patients in group B required atropine for bradycardia (Table 11).

\begin{tabular}{|c|c|c|c|c|}
\hline \multicolumn{2}{|c|}{ Variables } & $\begin{array}{c}\text { Group N } \\
\mathbf{N = 3 0}\end{array}$ & $\begin{array}{c}\text { Group C } \\
\mathbf{N = 3 0}\end{array}$ & $\begin{array}{c}\text { Group B } \\
\mathbf{N = 3 0}\end{array}$ \\
\hline \multicolumn{2}{|c|}{$\begin{array}{c}\text { Age } \\
\text { (Mean \& SD) }\end{array}$} & $47.9 \pm 10.27$ & $49.76 \pm 8.93$ & $55.1 \pm 7.87$ \\
\hline \multirow{4}{*}{$\begin{array}{c}\text { Age Cate- } \\
\text { gories }\end{array}$} & $\begin{array}{c}<50 \\
\text { yrs. }\end{array}$ & 10 & 16 & 9 \\
\cline { 2 - 5 } & $51-60$ & 8 & 11 & 15 \\
\cline { 2 - 5 } & $\begin{array}{c}61 \& \\
\text { Above }\end{array}$ & 12 & 3 & 6 \\
\hline \multirow{2}{*}{ Sex } & Male & 21 & 21 & 19 \\
\cline { 2 - 5 } & Female & 9 & 9 & 11 \\
\hline \multirow{2}{*}{ Group }
\end{tabular}

Group N= Normotensive group, C= Calcium channel blocker group, $\mathrm{B}=$ Beta blocker group

Data expressed by Mean Standard deviation and Number Table 1: Demographic Data

\begin{tabular}{|c|c|c|c|c|}
\hline \multicolumn{2}{|c|}{ Variables } & $\begin{array}{c}\text { Group N } \\
\mathbf{N = 3 0}\end{array}$ & $\begin{array}{c}\text { Group C } \\
\mathbf{N = 3 0}\end{array}$ & $\begin{array}{c}\text { Group B } \\
\mathbf{N = 3 0}\end{array}$ \\
\hline \multirow{2}{*}{$\begin{array}{c}\text { Block } \\
\text { height }\end{array}$} & $\mathrm{T}_{6}$ & 10 & 8 & 4 \\
\cline { 2 - 5 } & $\mathrm{T}_{8}$ & 14 & 12 & 4 \\
\cline { 2 - 5 } & $\mathrm{T}_{10}$ & 6 & 10 & 22 \\
\cline { 2 - 5 } & \multicolumn{4}{|c|}{ Average Level - T7 to T8 } \\
\hline \multicolumn{3}{|c|}{ Table 2: Sensory Levels in Different Groups } \\
\hline
\end{tabular}

\begin{tabular}{|c|c|c|c|}
\hline Variables & $\begin{array}{c}\text { Group N } \\
\mathbf{N}=\mathbf{3 0}\end{array}$ & $\begin{array}{c}\text { Group C } \\
\mathbf{N = 3 0}\end{array}$ & $\begin{array}{c}\text { Group B } \\
\mathbf{N = 3 0}\end{array}$ \\
\hline $\begin{array}{c}\text { Baseline } \\
\text { SAP } \\
\text { (mmHg) }\end{array}$ & $128.06 \pm 5.41$ & $134.53 \pm 5.42$ & $135.93 \pm 4.91$ \\
\hline $\begin{array}{c}\text { Baseline } \\
\text { DAP } \\
\text { (mmHg) }\end{array}$ & $76.07 \pm 7.03$ & $82.93 \pm 6.54$ & $80.73 \pm 9.23$ \\
\hline $\begin{array}{c}\text { Baseline } \\
\text { MAP } \\
\text { (mmHg) }\end{array}$ & $93.38 \pm 4.83$ & $100.18 \pm 5.95$ & $99.27 \pm 7.41$ \\
\hline $\begin{array}{c}\text { Baseline HR } \\
\text { (beats/min }\end{array}$ & $75.0 \pm 5.47$ & $72.46 \pm 5.81$ & $67.53 \pm 8.87$ \\
\hline \multicolumn{3}{|c|}{ Data expressed as Mean \pm SD } \\
\hline \multicolumn{3}{|c|}{ Table 3: Baseline Haemodynamics } \\
\hline
\end{tabular}

\begin{tabular}{|c|c|c|c|c|c|c|c|c|}
\hline & \multicolumn{2}{|c|}{ Group N } & \multicolumn{2}{|c|}{ Group C } & \multicolumn{2}{|c|}{ Group B } & \multicolumn{2}{|c|}{ Statistical Analysis } \\
\hline & $\begin{array}{c}\text { Mean } \\
\text { Difference }\end{array}$ & $\begin{array}{c}\text { Std. } \\
\text { Deviation } \\
\text { (SD) }\end{array}$ & $\begin{array}{c}\text { Mean } \\
\text { Difference }\end{array}$ & $\begin{array}{c}\text { Std. } \\
\text { Deviation }\end{array}$ & $\begin{array}{c}\text { Mean } \\
\text { Difference }\end{array}$ & $\begin{array}{c}\text { Std. } \\
\text { Deviation }\end{array}$ & $\begin{array}{c}\text { One } \\
\text { Way } \\
\text { ANOVA }\end{array}$ & $\begin{array}{c}\text { Significance } \\
\text { P }\end{array}$ \\
\hline Base \& 0 & 4.66 & 5.4 & 2.6 & 5.63 & 4.5 & 5.7 & 1.24 & Not Sig \\
\hline Base \& 2 min & 10.3 & 9.12 & 13.1 & 10.7 & 8.87 & 5.78 & 1.77 & Not Sig \\
\hline Base \& 4 min & 16 & 8.2 & 27 & 15 & 15 & 16 & 7.04 & $\mathrm{P}<0.001$ \\
\hline Base \& 6 min & 20.6 & 10.3 & 30.27 & 17.58 & 20.4 & 9.5 & 5.66 & $\mathrm{P}<0.005$ \\
\hline Base \& 8 min & 20 & 11 & 29 & 18 & 20 & 11 & 3.77 & $\mathrm{P}<0.02$ \\
\hline Base \& $10 \mathrm{~min}$ & 21 & 10 & 31 & 18 & 21 & 9.9 & 5.60 & $\mathrm{P}<0.005$ \\
\hline Base \& $15 \mathrm{~min}$ & 17.8 & 10.4 & 27 & 16.95 & 23.3 & 6.8 & 2.23 & Not Sig \\
\hline
\end{tabular}

\begin{tabular}{|c|c|c|c|}
\hline & Normotensive Vs Calcium Channel & Normotensive Vs Beta Blocker & \multirow{2}{*}{ Calcium Channel Vs Beta Blocker } \\
\hline & $0.60, \mathrm{NS}$ & $1.0, \mathrm{NS}$ & $0.46, \mathrm{NS}$ \\
\hline Base \& 0 & $0.23, \mathrm{NS}$ & $1.0, \mathrm{NS}$ & $0.66, \mathrm{NS}$ \\
\hline Base \& 2 min & $\mathrm{P}<0.004$ & $1.0, \mathrm{NS}$ & $\mathrm{P}<0.006$ \\
\hline Base \& 4 min & $\mathrm{P}<0.01$ & $1.0, \mathrm{NS}$ & $\mathrm{P}<0.04$ \\
\hline Base \& 6 min & $0.07, \mathrm{NS}$ & $1.0, \mathrm{NS}$ \\
\hline Base \& 8 min & $\mathrm{P}, 0.01$ & $1.0, \mathrm{NS}$ & $\mathrm{P}<0.01$ \\
\hline Base \& 10 min & $1, \mathrm{NS} \quad 0.23, \mathrm{NS}$ & $0.19, \mathrm{NS}$ \\
\hline Base \& 15 min & 'P' value significant between Group N and Group C \& Group B and Group C \\
\hline
\end{tabular}




\begin{tabular}{|c|c|c|c|c|c|c|c|c|}
\hline & \multicolumn{2}{|c|}{ Group N } & \multicolumn{2}{|c|}{ Group C } & \multicolumn{2}{|c|}{ Group B } & \multicolumn{2}{|c|}{ Statistical Analysis } \\
\hline & $\begin{array}{c}\text { Mean } \\
\text { Difference }\end{array}$ & $\begin{array}{c}\text { Std } \\
\text { Deviation }\end{array}$ & $\begin{array}{c}\text { Mean } \\
\text { Difference }\end{array}$ & $\begin{array}{c}\text { Std } \\
\text { Deviation }\end{array}$ & $\begin{array}{c}\text { Mean } \\
\text { Difference }\end{array}$ & $\begin{array}{c}\text { Std } \\
\text { Deviation }\end{array}$ & $\begin{array}{c}\text { One } \\
\text { Way } \\
\text { ANOVA }\end{array}$ & Significance \\
\hline Base \& 0 & 1.5 & 0.4 & 3 & 2 & 2.1 & 1.3 & .58 & Not Sig \\
\hline Base $\& 2$ min & 4.6 & 0.48 & 11.27 & 0.4 & 8.33 & 0.48 & 7.04 & $\mathrm{P}<0.001$ \\
\hline Base \& 4 min & 8.93 & 1.1 & 21.2 & 4.5 & 13.6 & 2.3 & 10.31 & $\mathrm{P}<0.000$ \\
\hline Base \& 6 min & 14.5 & 4.9 & 23.3 & 4.1 & 14.7 & 2.8 & 4.94 & $\mathrm{P}<0.009$ \\
\hline Base \& 8 min & 14.5 & 4.9 & 22.6 & 4.18 & 16.4 & 1.79 & 4.13 & $\mathrm{P}<0.01$ \\
\hline Base \& $10 \mathrm{~min}$ & 13.47 & 2.88 & 23.4 & 6.37 & 17.13 & 0.21 & 7.39 & $\mathrm{P}<0.001$ \\
\hline Base \& $15 \mathrm{~min}$ & 13.42 & 2.78 & 22.7 & 6.17 & 17.03 & 0.2 & 7.22 & $\mathrm{P}<0.01$ \\
\hline
\end{tabular}

Drop in DBP is expressed as Mean \pm SD and statistically significant throughout

Table 6: Diastolic Blood Pressure (DBP) Change After Spinal Anaesthesia (SA)

Bonferroni Multiple Comparisons

\begin{tabular}{|c|c|c|c|}
\hline \multicolumn{4}{|c|}{ Bonferroni Multiple Comparisons } \\
\hline & $\begin{array}{l}\text { Normotensive Vs } \\
\text { Calcium Channel }\end{array}$ & $\begin{array}{l}\text { Normotensive Vs } \\
\text { Beta Blocker }\end{array}$ & $\begin{array}{c}\text { Calcium Channel Vs } \\
\text { Beta Blocker }\end{array}$ \\
\hline Base \& 0 & NS & NS & NS \\
\hline Base $\& 2 \mathrm{~min}$ & $\mathrm{P}<0.02$ & NS & $\mathrm{P}<0.001$ \\
\hline Base \& 4 min & $\mathrm{P}<0.02$ & NS & $\mathrm{P}<0.000$ \\
\hline Base \& 6 min & $\mathrm{P}<0.02$ & NS & $\mathrm{P}<0.02$ \\
\hline Base \& 8 min & $\mathrm{P}<0.02$ & NS & $\mathrm{P}<0.02$ \\
\hline Base \& $10 \mathrm{~min}$ & $\mathrm{P}<0.05$ & NS & $\mathrm{P}<0.001$ \\
\hline Base \& $15 \mathrm{~min}$ & $\mathrm{P}<0.05$ & NS & $\mathrm{P}<0.001$ \\
\hline \multicolumn{4}{|c|}{ P value is insignificant between Group N and Group B and Group C and Group B } \\
\hline \multicolumn{4}{|c|}{ Table 7: Intergroup Comparison of ' $P^{\prime}$ Values in DBP } \\
\hline
\end{tabular}

\begin{tabular}{|c|c|c|c|c|c|c|c|c|}
\hline & \multicolumn{2}{|c|}{ Group N } & \multicolumn{2}{|c|}{ Group C } & \multicolumn{2}{|c|}{ Group B } & \multicolumn{2}{|c|}{ Statistical Analysis } \\
\hline & $\begin{array}{c}\text { Mean } \\
\text { Difference }\end{array}$ & $\begin{array}{c}\text { Std. } \\
\text { Deviation }\end{array}$ & $\begin{array}{c}\text { Mean } \\
\text { Difference }\end{array}$ & $\begin{array}{c}\text { Std. } \\
\text { Deviation }\end{array}$ & $\begin{array}{c}\text { Mean } \\
\text { Difference }\end{array}$ & $\begin{array}{c}\text { Std. } \\
\text { Deviation }\end{array}$ & $\begin{array}{c}\text { One } \\
\text { Way } \\
\text { ANOVA }\end{array}$ & Significance \\
\hline Base \& 0 & 3.7 & 0.9 & 2.8 & 1 & 3.1 & 1.1 & .36 & Not Sig \\
\hline Base \& 2 min & 7.82 & 0.63 & 11.94 & 1.06 & 8.6 & 0.75 & 3.54 & $\mathrm{P}<0.03$ \\
\hline Base \& 4 min & 7.83 & 0.8 & 23.11 & 6.3 & 14.4 & 3.18 & 10.11 & $\mathrm{P}<0.000$ \\
\hline Base \& 6 min & 11.9 & 0.87 & 25.73 & 6.36 & 16.74 & 2.35 & 5.31 & $\mathrm{P}<0.007$ \\
\hline Base \& $8 \mathrm{~min}$ & 18.32 & 5.38 & 25 & 6 & 18 & 3 & 2.98 & $\mathrm{P}<0.05$ \\
\hline Base \& $10 \mathrm{~min}$ & 18 & 6 & 26 & 8 & 18 & 1 & 6.97 & $\mathrm{P}<0.002$ \\
\hline Base \& $15 \mathrm{~min}$ & 17.9 & 6 & 25.83 & 6 & 17.8 & 1 & 6.96 & $\mathrm{P}<0.002$ \\
\hline
\end{tabular}

\begin{tabular}{|c|c|c|c|}
\hline \multicolumn{4}{|c|}{ Bonferroni Multiple Comparisons } \\
\hline & $\begin{array}{l}\text { Normotensive Vs } \\
\text { Calcium Channel }\end{array}$ & $\begin{array}{l}\text { Normotensive Vs } \\
\text { Beta Blocker }\end{array}$ & $\begin{array}{c}\text { Calcium Channel Vs } \\
\text { Beta Blocker }\end{array}$ \\
\hline Base \& 0 & Ns & NS & Ns \\
\hline Base \& 2 min & & NS & $\mathrm{P}<0.02$ \\
\hline Base \& 4 min & $\mathrm{P}<0.004$ & $\mathrm{P}<0.02$ & $\mathrm{P}<0.000$ \\
\hline Base \& 6 min & $\mathrm{P}<0.009$ & NS & $P<0.04$ \\
\hline Base \& $8 \mathrm{~min}$ & $P<0.009$ & NS & NS \\
\hline Base \& $10 \mathrm{~min}$ & $\mathrm{P}<0.01$ & NS & $\mathrm{P}<0.02$ \\
\hline Base \& 15 min & $\mathrm{P}<0.01$ & NS & $\mathrm{P}<0.02$ \\
\hline & e 9: Intergro & Values for $M A$ & \\
\hline
\end{tabular}

\begin{tabular}{|c|c|c|c|c|c|c|c|c|}
\hline & \multicolumn{2}{|c|}{ Group N } & \multicolumn{2}{|c|}{ Group C } & \multicolumn{2}{|c|}{ Group B } & \multicolumn{2}{|c|}{ Statistical Analysis } \\
\hline & $\begin{array}{c}\text { Mean } \\
\text { Difference }\end{array}$ & $\begin{array}{c}\text { Std. } \\
\text { Deviation }\end{array}$ & $\begin{array}{c}\text { Mean } \\
\text { Difference }\end{array}$ & $\begin{array}{c}\text { Std. } \\
\text { Deviation }\end{array}$ & $\begin{array}{c}\text { Mean } \\
\text { Difference }\end{array}$ & $\begin{array}{c}\text { Std. } \\
\text { Deviation }\end{array}$ & $\begin{array}{c}\text { One Way } \\
\text { ANOVA }\end{array}$ & Significance \\
\hline Base \& 0 & 0.4 & 1.4 & 0.2 & 0.1 & 3.53 & 2.28 & 7.53 & $\mathrm{P}<0.001$ \\
\hline Base \& 2 min & 1.93 & 1.9 & 0.73 & 0.1 & 5.2 & 2.4 & 6.15 & $\mathrm{P}<0.003$ \\
\hline Base \& 4 min & 2.66 & 1.28 & 1 & 0.14 & 6.5 & 1.8 & 10.98 & $\mathrm{P}<0.000$ \\
\hline Base \& 6 min & 3.2 & 2 & 2.3 & 0.82 & 7.7 & 1.7 & 9.20 & $\mathrm{P}<0.000$ \\
\hline Base \& $8 \mathrm{~min}$ & 2.4 & 2 & 3.2 & 0.9 & 10.2 & 0.58 & 14.16 & $\mathrm{P}<0.000$ \\
\hline Base \& $10 \mathrm{~min}$ & 2.5 & 2.3 & 2.67 & 0.52 & 10.4 & 0.81 & 17.05 & $\mathrm{P}<0.000$ \\
\hline Base \& $15 \mathrm{~min}$ & 2.4 & 2.2 & 2.7 & 0.54 & 10.5 & 0.8 & 16.84 & $\mathrm{P}<0.000$ \\
\hline
\end{tabular}




\begin{tabular}{|c|c|c|c|}
\hline \multicolumn{4}{|c|}{ Bonferroni Multiple Comparisons } \\
\hline & $\begin{array}{c}\text { Normotensive Vs } \\
\text { Calcium Channel }\end{array}$ & $\begin{array}{c}\text { Normotensi } \\
\text { ve Vs } \\
\text { Beta } \\
\text { Blocker }\end{array}$ & $\begin{array}{c}\text { Calcium } \\
\text { Channel Vs } \\
\text { Beta Blocker }\end{array}$ \\
\hline Base \& 0 & $\mathrm{P}<0.005$ & $\mathrm{P}<0.003$ & $\mathrm{P}<0.005$ \\
\hline $\begin{array}{c}\text { Base \& } \\
\text { min }\end{array}$ & NS & $\mathrm{P}<0.04$ & $\mathrm{P}<0.003$ \\
\hline $\begin{array}{c}\text { Base \& 4 } \\
\text { min }\end{array}$ & NS & $\mathrm{P}<0.006$ & $\mathrm{P}<0.000$ \\
\hline $\begin{array}{c}\text { Base \& 6 } \\
\text { min }\end{array}$ & NS & $\mathrm{P}<0.004$ & $\mathrm{P}<0.000$ \\
\hline $\begin{array}{c}\text { Base \& 8 } \\
\text { min }\end{array}$ & NS & $\mathrm{P}<0.000$ & $\mathrm{P}<0.000$ \\
\hline $\begin{array}{c}\text { Base \& 10 } \\
\text { min }\end{array}$ & NS & $\mathrm{P}<0.000$ & $\mathrm{P}<0.000$ \\
\hline $\begin{array}{c}\text { Base \& 15 } \\
\text { min }\end{array}$ & NS & $\mathrm{P}<0.000$ & $\mathrm{P}<0.000$ \\
\hline \multicolumn{2}{|c|}{ 'P' value is significant between Group N \& Group B and also } \\
between Group C and Group B \\
\hline \multicolumn{4}{|c|}{ Table 11: Intergroup Comparison of P Values for PR } \\
\hline
\end{tabular}

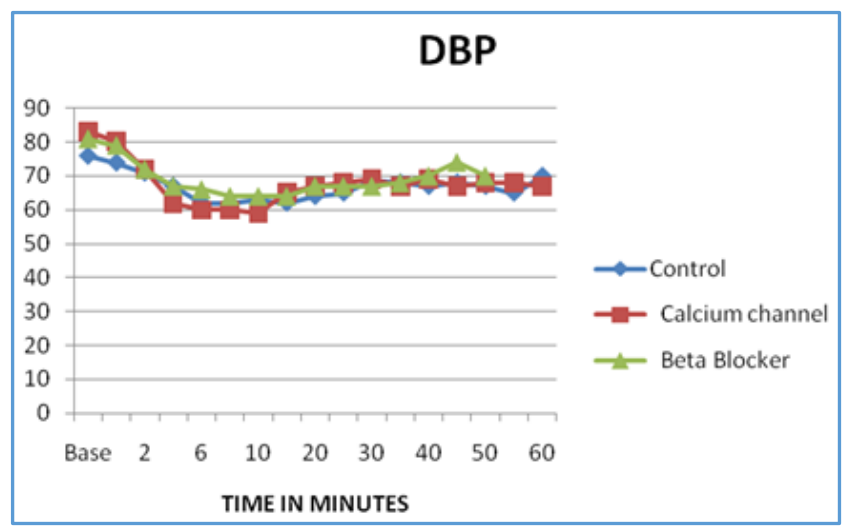

Fig. 2: Diastolic Blood Pressure Changes

$\mathrm{X}$-axis - Time, $\mathrm{Y}$ axis - DBP in mmHg. The maximum fall of DBP is seen at 8-10 min in both the groups.

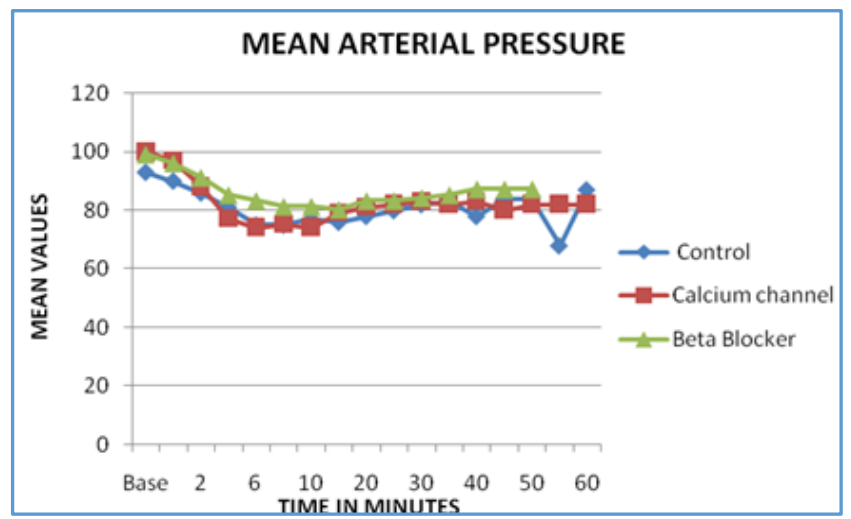

Fig. 3: Mean Arterial Pressure Changes

$\mathrm{X}$-axis -time, $\mathrm{Y}$ axis -mean arterial pressure in $\mathrm{mmHg}$. The maximum fall of MAP is seen at $8 \mathrm{~min}$ in both the groups.

\section{DISCUSSION}

Hypotension is the most frequent side effect associated with spinal anaesthesia, predisposing the individual to myocardial and brain ischaemia. Identification of risk factors for prediction of early hypotension were age, female sex, body mass index $>30 \mathrm{~kg} / \mathrm{m} 2$, history of hypertension, diabetes mellitus, anaemia, high baseline heart rate, high systolic and diastolic blood pressure, pulse pressure, sensory level of blockade higher than or equal to T6. Association of hypotension was more in patients with $\mathrm{h}$ /o hypertension (OR -1.739) and incidence reduced in patients on antihypertensives (1.012). ${ }^{1}$

Risk factors must be easily obtainable and known before the blockade to meet their preventive aim..$^{2}$ Alek Rook et $\mathrm{al}^{3}$ found out that an exaggerated decrease in blood pressure occurs in elderly patients with cardiac disease and hypotension was mainly a result of decrease in systemic vascular resistance. Hence, the knowledge of risk factors for exaggerated hypotension after spinal anaesthesia may help the anaesthesiologist in adopting preventive measures to minimize the catastrophe. We chose and compared the haemodynamic changes in hypertensive patients without any other cardiac morbidity on antihypertensive drugs for $>1$ month duration with that of normotensives. $\mathrm{mmHg}$. The maximum fall of SBP is seen at $8 \mathrm{~min}$ in both the groups. 
Our study compared standardized doses of calcium channel blocker (Amlodipine $10 \mathrm{mg}$ ) (Group C), B blocker (Atenolol $50 \mathrm{mg}$ ) (Group B) on Haemodynamics after spinal anaesthesia with normotensive patients since the effect on cardiac and vasculature is dose dependent and previous study.4 had shown effect of different doses of antihypertensives within the group on haemodynamics. But our study observations also correlated well with this study, which showed that hypotension is more common in patients who are on CCBs (Group C) (18/30) and $80 \%$ of them required fluids and vasopressors more than those on B blocker or normal patients, effect could be due to peripheral vasodilatation already existing in pts on CCBs and could have exaggerated by decreased vascular resistance after spinal anaesthesia.

Studies have been done on effect of B blocker and CCB during induction of general anaesthesia. ${ }^{6}$, effect of the same on laryngoscopy and intubation. ${ }^{5}$ hypotension during induction is primarily due to decrease in SVR, which is also one of the primary mechanism with sympathetic blockade after spinal and effect of ACE I on post spinal blood pressure. ${ }^{7}$ and found hypotension $>25 \%$ can be detrimental to vital organ circulation.

Mojica JL et $\mathrm{al}^{8}$ have studied regarding the timing of IV fluids during spinal anaesthesia and found coloading (IV fluids during spinal anaesthesia) is more advantageous than preloading in prevention of severe hypotension or Arndlt JO et $\mathrm{al}^{9}$ compared effectiveness of fluids and prophylactic vasopressors in prevention of severe hypotension. We used 15 $\mathrm{mL} / \mathrm{kg}$ of Ringer's lactate to reason out hypotension was mainly the effect of vasodilatation after spinal and precipitated effect of antihypertensives and not due to absence of coloading.

Parida S.10 described in case series regarding the severity of Hypotension following spinal anaesthesia in patients on amlodipine $10 \mathrm{mg}$ even after withholding morning dose and preloading before spinal, our study correlated with the findings of the series but degree of hypotension was less probably because study group involved young patients also where the vasomotor tone is preserved compared to older individuals. ${ }^{11}$

Structural changes (Medial hyperplasia and hypertrophy) in arteriolar walls play a primary role in haemodynamic response to anaesthesia and explain greater changes in systemic vascular resistance and arterial pressure in hypertensive patients than normotensive patients with similar degree of sympathetic blockade. ${ }^{12}$ In Singla et al ${ }^{1}$ study there were patients not taking any treatment for hypertension, they all fell in hypotensive group. Regular antihypertensive treatment was associated with reduced risk of hypotension. Prolonged antihypertensive treatment may induce regression of the structural changes in arterioles and consequently a more normal functional response to vasodilatation or constriction.

Vukalic J.13 et al studied elderly hypertensives on B blocker medication and found continuation of B blockers in the pre-operative period was associated with hypotension, bradycardia requiring atropine and nausea after spinal anaesthesia compared to other group which discontinued the drug, so they concluded discontinuation of B blockers in preoperative period is prudent, but other studies. ${ }^{14}$ however found that cessation of beta blocker therapy leads to rebound hypertension, arrhythmias and this followed by various cardiovascular consequences, it is beneficial to continue the $\mathrm{B}$ blockers pre-operatively. Our study correlated with the above study except lesser degree of hypotension, which could be due to inclusion of young individuals in the study even after continuation of B blockers, but bradycardia was significant $(16 / 30)$ requiring atropine in all patients.

\section{CONCLUSION}

Our study showed exaggerated hypotension in patients on calcium channel blockers, which could be partly explained by the vasodilator property of the drug which could have additive effect after spinal anaesthesia inducing exaggerated hypotension. This exaggerated fall in blood pressure after spinal anaesthesia warrants the discontinuation of calcium channel blockers before surgery. Bradycardia following spinal anaesthesia was entirely limited to the beta blocker group in our study. The knowledge of these risk factors could be useful in increasing vigilance in those patients most at risk for hypotension, in allowing timely therapeutic intervention and taking preventive measure to minimize the spinal hypotension.

\section{REFERENCES}

1. Singla D, Kathuria S, Singh A, et al. Risk factors for development of early hypotension during spinal anaesthesia. J Anaesthesiol Clin Pharmacol 2006;22(4):387-93.

2. Hartmann B, Junger A, Klasen J, et al. The incidence and risk factors for hypotension after spinal anaesthesia induction: an analysis with automated data collection. Anaesth Analg 2002;94(6):1521-9.

3. Rooke GA, Freund PR, Jacobson AF. Haemodynamic response and change in organ blood volume during spinal anaesthesia in elderly men with cardiac disease. Anaesth Analg 1997;85(1):99-105.

4. Kaimar P, Sanji N, Upadya M, et al. A comparison of hypotension and bradycardia following spinal anaesthesia in patients on calcium channel blockers and beta-blockers. Indian journal of pharmacology 2012;44(2):193-6.

5. Swear JW, Jewkes C, Tellez JC, et al. Does the choice of antihypertensive therapy influence haemodynamic responses to induction, laryngoscopy and intubation. British Journal of Anaesthesia 1994;73(3):303-8.

6. Samad K, Khan F, Azam I. Haemodynamic effects of anaesthetic induction in patients treated with beta and calcium channel blockers. Middle East J Anaesthesiol 2008;19(5):1111-28.

7. Hohne C, Meier L, Boemke W, et al. Angiotensin convertase inhibitor inhibitors do not exaggerate the blood pressure decrease in the early phase of spinal anaesthesia. Acta Anaesthesiologica Scandinavica 2003;47(7):891-6.

8. Mojica JL, Meléndez HJ, Bautista LE. The timing of intravenous crystalloid administration and incidence of cardiovascular side effects during spinal anaesthesia: the results from a randomized controlled trial. Anaesth Analg 2002;94(2):432-7.

9. Arndt J0, Bömer W, Krauth J, et al. Incidence and time course of cardiovascular side effects during spinal anaesthesia after prophylactic administration of 
intravenous fluids or vasoconstrictors. Anaesth Analg 1998;87(2):347-54.

10. Parida S, Nawaz M, Kundra P. Severe hypotension following spinal anaesthesia in patients on amlodipine. J Anaesthesiol Clin Pharmacol 2012;28(3):408-9.

11. Alecu C, Cuignet-Royer E, Mertes PM, et al. Pre-existing arterial stiffness can predict hypotension during induction of anaesthesia in the elderly. Br J Anaesth 2010;105(5):583-8.
12. Folkow B. Cardiovascular structural adaptation : its role in the initiation and maintenance of primary hypertension. Clin Sci 1978;55:3s-22s.

13. Vukalic J, Rakaric MP. Adverse effects of beta adrenergic blockers during spinal anaesthesia with $0.5 \%$ bupivacaine. Crit Care 2010;14(Suppl 1):P480.

14. Ponten J, Biber B, Bjuro T, et al. Beta receptor blockade and spinal anaesthesia. Withdrawal versus continuation of long term therapy. Acta Anaesthesiol Scand Suppl 1982;76:62-9. 